\title{
THE GENERAL TOTALLY POSITIVE MATRIX COMPLETION PROBLEM WITH FEW UNSPECIFIED ENTRIES*
}

\author{
SHAUN M. FALLAT ${ }^{\dagger}$, CHARLES R. JOHNSON ${ }^{\ddagger}$, AND RONALD L. SMITH ${ }^{\S}$
}

\begin{abstract}
For $m$-by- $n$ partial totally positive matrices with exactly one unspecified entry, the set of positions for that entry that guarantee completability to a totally positive matrix are characterized. They are the positions $(i, j), i+j \leq 4$ and the positions $(i, j), i+j \geq m+n-2$. In each case, the set of completing entries is an open (and infinite in case $i=j=1$ or $i=m, j=n$ ) interval. In the process some new structural results about totally positive matrices are developed. In addition, the pairs of positions that guarantee completability in partial totally positive matrices with two unspecified entries are characterized in low dimensions.
\end{abstract}

Key words. totally positive matrices, matrix completion problems, partial matrix.

AMS subject classifications. 15A48

1. Introduction. An $m$-by- $n$ matrix $A$ is called totally positive, TP, (totally nonnegative, TN,) if every minor of $A$ is positive (nonnegative). A partial matrix is a rectangular array in which some entries are specified, while the remaining, unspecified, entries are free to be chosen from an indicated set (e.g., the real field). A completion of a partial matrix is a choice of allowed values for the unspecified entries, resulting in a conventional matrix, and a matrix completion problem asks which partial matrices have a completion with a particular desired property. The TP, TN matrices arise in a variety of ways in geometry, combinatorics, algebra, differential equations, function theory, etc., and have attracted considerable attention recently $[1,7,9,17]$. There is also now considerable literature on a variety of matrix completion problems $[3,4,5,6,8,12,13,14,15]$. Since total positivity and total nonnegativity are inherited by arbitrary submatrices, in order for a partial matrix to have a TP (TN) completion, it must be partially $\mathrm{TP}(\mathrm{TN})$, i.e., every fully specified submatrix must be TP (TN). Study of the TN completion problem was begun in [14], where the square, combinatorially symmetric patterns for which every partially TN matrix has a TN completion were characterized under a regularity condition (that has been removed in $[6])$.

Here, we begin the study of the TP completion problem, in the rectangular case

\footnotetext{
${ }^{*}$ Received by the editors on 13 July 1999. Accepted for publication on 10 January 2000. Handling Editor: Ludwig Elsner.

†Department of Mathematics and Statistics, University of Regina, Regina, Saskatchewan, S4S 0A2, CANADA. The work of this author was begun while as a Ph.D candidate at the College of William and Mary (sfallat@math.uregina.ca).

$\ddagger$ Department of Mathematics, College of William and Mary, Williamsburg, VA. 23187-8795, USA (crjohnso@math.wm.edu). Research supported in part by the Office of Navel Research contract N00014-90-J-1739 and NSF grant 92-00899.

$\S$ Department of Mathematics, University of Tennessee at Chattanooga, Chattanooga, TN 374032598, USA (rsmith@cecasun.utc.edu). The work of this author was begun while on sabbatical at the College of William and Mary, supported by the University of Chattanooga Foundation. The research was completed under a Summer Research grant from the University of Tennessee at Chattanooga's Center of Excellence in Computer Applications.
} 
without any combinatorial symmetry assumption. It should be noted that there are important differences between the TP and TN completion problems. In the former, there are stronger requirements on the data, but also much stronger requirements on the completion. In practice, this difference is considerable, and for example, results analogous to those in [14] are not yet clear in the TP case. Here in the TP case we again focus on patterns, and ask for which patterns does every partial TP matrix have a TP completion. In general, this appears to be a substantial and subtle problem. We give a complete answer for $m$-by- $n$ patterns with just one unspecified entry, and even here the answer is rather surprising and nontrivial. If $\min \{m, n\} \geq 4$, then only 12 individual positions ensure completability: the upper left and lower right triangles of 6 entries each. If $\min \{m, n\}=1,2$ or 3 , then any single position ensures completability. In order to prove these results several classical and some new (e.g., about TP linear systems) facts about TP matrices are used. Patterns with two unspecified entries are also considered. An inventory of completable pairs when $m, n \leq 4$, and some general observations are given. However, the general question of two unspecified entries is shown to be subtle: examples are given to show that the answer does not depend just on the "relative" positions of the two entries.

The submatrix of an $m$-by- $n$ matrix $A$ lying in rows $\alpha$ and columns $\beta, \alpha \subseteq M=$ $\{1,2 \ldots, m\}, \beta \subseteq N=\{1,2 \ldots, n\}$, is denoted by $A[\alpha, \beta]$. Similarly, $A(\alpha, \beta)$ denotes the submatrix obtained from $A$ by deleting the rows indexed by $\alpha$ and columns indexed by $\beta$. When $m=n$, the principal submatrix $A[\alpha, \alpha]$ is abbreviated to $A[\alpha]$, and the complementary principal submatrix is denoted by $A(\alpha)$. As usual, for $\alpha \subseteq N$ we let $\alpha^{c}$ denote the complement of $\alpha$ relative to $N$. An $m$-by- $n$ matrix is $\mathrm{TN}_{k}\left(\right.$ resp. $\left.\mathrm{TP}_{k}\right)$ for $1 \leq k \leq \min (m, n)$, if all minors of size at most $k$ are nonnegative (resp. positive). It is well known that if $A=\left[a_{i j}\right]$ is an $m$-by- $n \mathrm{TP}_{k}\left(\mathrm{TN}_{k}\right)$ matrix, then the $m$-by- $n$ matrix defined by $\tilde{A}=\left[a_{m-i+1, n-j+1}\right]$ is also a $\mathrm{TP}_{k}\left(\mathrm{TN}_{k}\right)$ matrix. We may refer to the matrix $\tilde{A}=\left[a_{m-i+1, n-j+1}\right]$ as being obtained from $A$ by reversing the indices of A.

We now present some identities, discovered by Sylvester (see [10]), for completeness and clarity of composition. Let $A$ be an $n$-by- $n$ matrix, $\alpha \subseteq N$, and suppose $|\alpha|=k$. Define the $(n-k)$-by- $(n-k)$ matrix $B=\left[b_{i j}\right]$, with $i, j \in \alpha^{c}$, by setting $b_{i j}=\operatorname{det} A[\alpha \cup\{i\}, \alpha \cup\{j\}]$, for every $i, j \in \alpha^{c}$. Then Sylvester's identity states that for each $\delta, \gamma \subset \alpha^{c}$, with $|\delta|=|\gamma|=l$,

$$
\operatorname{det} B[\delta, \gamma]=(\operatorname{det} A[\alpha])^{l-1} \operatorname{det} A[\alpha \cup \delta, \alpha \cup \gamma] .
$$

Observe that a special case of $(1)$ is $\operatorname{det} B=(\operatorname{det} A[\alpha])^{n-k-1} \operatorname{det} A$. Another very useful special case which is employed throughout is the following. Let $A$ be an $n$-by- $n$ matrix partitioned as follows

$$
A=\left[\begin{array}{ccc}
a_{11} & a_{12}^{T} & a_{13} \\
a_{21} & A_{22} & a_{23} \\
a_{31} & a_{32}^{T} & a_{33}
\end{array}\right],
$$

where $A_{22}$ is $(n-2)$-by- $(n-2)$ and $a_{11}, a_{33}$ are scalars. Define the matrices 


$$
\begin{aligned}
& B=\left[\begin{array}{ll}
a_{11} & a_{12}^{T} \\
a_{21} & A_{22}
\end{array}\right], \quad C=\left[\begin{array}{ll}
a_{12}^{T} & a_{13} \\
A_{22} & a_{23}
\end{array}\right], \\
& D=\left[\begin{array}{ll}
a_{21} & A_{22} \\
a_{31} & a_{32}^{T}
\end{array}\right], \quad E=\left[\begin{array}{ll}
A_{22} & a_{23} \\
a_{32}^{T} & a_{33}
\end{array}\right] .
\end{aligned}
$$

If we let $\tilde{b}=\operatorname{det} B, \tilde{c}=\operatorname{det} C, \tilde{d}=\operatorname{det} D$, and $\tilde{e}=\operatorname{det} E$, then by (1) it follows that

$$
\operatorname{det}\left[\begin{array}{cc}
\tilde{b} & \tilde{c} \\
\tilde{d} & \tilde{e}
\end{array}\right]=\operatorname{det} A_{22} \operatorname{det} A .
$$

Hence, provided $\operatorname{det} A_{22} \neq 0$, we have

$$
\operatorname{det} A=\frac{\operatorname{det} B \operatorname{det} E-\operatorname{det} C \operatorname{det} D}{\operatorname{det} A_{22}} .
$$

We now state an important result due to Fekete (see, e.g., [1]), who gives a very useful and appealing criterion (or characterization) of totally positive matrices. First we need to define the notion of the dispersion of an index set. For $\alpha=\left\{i_{1}, i_{2}, \ldots, i_{k}\right\} \subseteq$ $N$, with $i_{1}<i_{2}<\cdots<i_{k}$, the dispersion of $\alpha$, denoted by $d(\alpha)$, is defined to be $\sum_{j=1}^{k-1}\left(i_{j+1}-i_{j}-1\right)=i_{k}-i_{1}-(k-1)$, with the convention that $d(\alpha)=0$ when $\alpha$ is a singleton. The dispersion of a set $\alpha$ represents a measure of the "gaps" in the set $\alpha$. In particular, observe that $d(\alpha)=0$ whenever $\alpha$ is a contiguous (i.e., an index set based on consecutive indices) subset of $N$.

TheOREm 1.1. (Fekete's Criterion) An m-by-n matrix $A$ is totally positive if and only if $\operatorname{det} A[\alpha, \beta]>0$, for all $\alpha \subseteq\{1,2, \ldots, m\}$ and $\beta \subseteq\{1,2, \ldots, n\}$, with $|\alpha|=|\beta|$, and $d(\alpha)=d(\beta)=0$. In other words a matrix is totally positive if and only if the determinant of every square submatrix based on contiguous row and column index sets is positive.

The next lemma (which may be of independent interest) proves to be very useful for certain completion problems for TP matrices, and is no doubt useful for other issues with TP matrices.

Lemma 1.2. Let $A=\left[a_{1}, a_{2} \cdots, a_{n}\right]$ be an $(n-1)$-by-n TP matrix. Then, for $k=1,2, \cdots, n$,

$$
a_{k}=\sum_{\substack{i=1 \\ 1 \neq k}}^{n} y_{i} a_{i},
$$

has a unique solution y for which

$$
\operatorname{sgn}\left(y_{i}\right)= \begin{cases}\operatorname{sgn}(-1)^{i}, & \text { if } k \text { is odd } \\ \operatorname{sgn}(-1)^{i-1}, & \text { if } k \text { is even }\end{cases}
$$


Proof. If $k=1$, (3) has solution

$$
\begin{aligned}
y & =\left[a_{2}, a_{3}, \cdots, a_{n}\right]^{-1} a_{1} \\
& =\frac{1}{\operatorname{det}\left[a_{2}, a_{3}, \cdots, a_{n}\right]}\left[\begin{array}{c}
\operatorname{det}\left[a_{1}, a_{3}, a_{4}, \cdots, a_{n}\right] \\
\operatorname{det}\left[a_{2}, a_{1}, a_{4}, \cdots, a_{n}\right] \\
\vdots \\
\operatorname{det}\left[a_{2}, a_{3}, \cdots, a_{n-1}, a_{1}\right]
\end{array}\right]
\end{aligned}
$$

and $\operatorname{sgn}\left(y_{i}\right)=\operatorname{sgn}(-1)^{i}$. If $k>1$, then (3) has solution

$$
\begin{gathered}
y=\left[a_{1}, a_{2}, \cdots, a_{k-1}, a_{k+1}, \cdots, a_{n}\right]^{-1} a_{k} \\
=\frac{1}{\operatorname{det}\left[a_{1}, \cdots, a_{k-1}, a_{k+1}, \cdots, a_{n}\right]}\left[\begin{array}{c}
\operatorname{det}\left[a_{k}, a_{2}, a_{3}, \cdots, a_{k-1}, a_{k+1}, \cdots, a_{n}\right] \\
\operatorname{det}\left[a_{1}, a_{k}, a_{3}, \cdots, a_{k-1}, a_{k+1}, \cdots, a_{n}\right] \\
\vdots \\
\vdots \\
\operatorname{det}\left[a_{1}, a_{2}, \cdots, a_{k-2}, a_{k}, a_{k+1}, \cdots, a_{n}\right] \\
\operatorname{det}\left[a_{1}, a_{2}, \cdots, a_{k-1}, a_{k}, a_{k+2}, \cdots, a_{n}\right] \\
\vdots \\
\operatorname{det}\left[a_{1}, a_{2}, \cdots, a_{k-1}, a_{k+1}, a_{k}, a_{k+3}, \cdots, a_{n}\right] \\
\vdots \\
\operatorname{det}\left[a_{1}, a_{2}, \cdots, a_{k-1}, a_{k+1}, \cdots, a_{n-1}, a_{k}\right]
\end{array}\right]
\end{gathered}
$$

and we see that if $k$ is odd, $\operatorname{sgn}\left(y_{i}\right)=\operatorname{sgn}(-1)^{i}$ while if $k$ is even, $\operatorname{sgn}\left(y_{i}\right)=$ $\operatorname{sgn}(-1)^{i-1}$.

2. Main Results. In this section we consider the TP completion problem for $m$-by- $n$ patterns with just one unspecified entry. We begin with a preliminary lemma which is used throughout the remainder of the paper.

Lemma 2.1. Let

$$
A=\left[\begin{array}{cccc}
x & a_{12} & \cdots & a_{1 n} \\
a_{21} & a_{22} & \cdots & a_{2 n} \\
\vdots & \vdots & & \vdots \\
a_{n 1} & a_{n 2} & \cdots & a_{n n}
\end{array}\right],
$$

$n \geq 2$, be partial TP in which $x$ is the only unspecified entry. Then, if $x$ is chosen so that $\operatorname{det} A \geq 0$, $\operatorname{det} A[\{1, \cdots, k\}]>0, k=1, \cdots, n-1$. (In particular, $x>0$.)

Proof. The proof follows inductively using Sylvester's identity (2) for determinants applied to the leading principal minors of $A$ in decreasing order of size. For example,

$$
\begin{aligned}
& 0 \leq \operatorname{det} A \operatorname{det} A[\{2,3, \ldots, n-1\}] \\
& =\operatorname{det} A[\{1,2, \ldots, n-1\}] \operatorname{det} A[\{2,3, \ldots, n\}] \\
& \quad-\operatorname{det} A[\{1,2, \ldots, n-1\},\{2, \ldots, n\}] \operatorname{det} A[\{2, \ldots, n\},\{1,2, \ldots, n-1\}],
\end{aligned}
$$


and since $A$ is partial TP we have that $\operatorname{det} A[\{1,2, \ldots, n-1\}]>0$. If $A$ is replaced by $A[\{1,2, \ldots, n-1\}]$ in the above identity, then it follows that $\operatorname{det} A[\{1,2, \ldots, n-2\}]>0$, etc. $\square$

THeOREM 2.2. Let $A$ be an $n$-by-n partial TP matrix, $n \geq 3$, with $x$, the only unspecified entry, lying in the $(s, t)$ position. If $s+t \leq 4$ or $s+t \geq 2 n-2$, then $A$ is completable to a TP matrix.

Proof. Throughout the proof $A=A(x)=A_{n}(x)$ will denote an $n$-by- $n$ partial TP matrix with exactly one unspecified entry $x$ in the $(s, t)$ position. For $k=1, \cdots, n-1$, let

$$
\begin{aligned}
& A_{k}(x)=A[\{1, \cdots, k\}], \\
& B_{k}(x)=A[\{1, \cdots, k\},\{2, \cdots, k+1\}], \\
& C_{k}(x)=A[\{2, \cdots, k+1\},\{1, \cdots, k\}], \text { and } \\
& D_{k}(x)=A[\{2, \cdots, k+1\}] .
\end{aligned}
$$

Case $1(s, t)=(1,1)$ : Every minor which does not involve $A[1,1]$ is positive by assumption. Furthermore $A[1,1]$ enters positively into every minor in which it is involved. Hence, by making $A[1,1]$ large enough, we will obtain a TP completion of A.

Case $2(s, t)=(1,2)$ : Let $F=A[\{2, \cdots, n-1\},\{2, \cdots, n\}]=\left[f_{1}, f_{2}, \cdots, f_{n-1}\right]$. By Lemma $1.2 f_{1}=\sum_{i=2}^{n-1} y_{i} f_{i}$ in which $\operatorname{sgn}\left(y_{i}\right)=\operatorname{sgn}(-1)^{i}$. Let $B_{n-1}\left(x_{B}\right)=$ $\left[b_{1}, b_{2}, \cdots, b_{n-1}\right]$ in which $x_{B}$ is chosen so that $b_{1}=\sum_{i=2}^{n-1} y_{i} b_{i}$. Thus, $\operatorname{det} B_{n-1}\left(x_{B}\right)=$ 0 and, by Lemma 2.1 , $\operatorname{det} B_{k}\left(x_{B}\right)>0, k=1,2, \cdots, n-2$; in particular, $x_{B}>0$. So

$$
\begin{aligned}
\operatorname{det} A_{n-1}\left(x_{B}\right) & =\operatorname{det}\left[b_{0}, b_{1}, b_{2}, \cdots, b_{n-2}\right] \\
& =\operatorname{det}\left[b_{0}, \sum_{i=2}^{n-1} y_{i} b_{i}, b_{2}, \cdots, b_{n-2}\right] \\
& =\operatorname{det}\left[b_{0}, y_{n-1} b_{n-1}, b_{2}, \cdots, b_{n-2}\right] \\
& =(-1)^{n-3} y_{n-1} \operatorname{det}\left[b_{0}, b_{2}, \cdots, b_{n-2}, b_{n-1}\right] \\
& =\left|y_{n-1}\right| \operatorname{det}\left[b_{0}, b_{2}, \cdots, b_{n-2}, b_{n-1}\right] \\
& >0 .
\end{aligned}
$$

Applying Sylvester's identity (2) for determinants, we obtain $\operatorname{det} A>0$ and we can continue to apply this identity to obtain:

$$
\operatorname{det} A_{k}\left(x_{B}\right)>0, k=2, \cdots, n-2 .
$$

We can then increase $x_{B}$ (so as to make $\operatorname{det} B_{n-1}(x)>0$ ) and obtain a TP completion of $A$.

Case $3(s, t)=(1,3)$ : Notice that it suffices to show that there is $x>0$ such that each of

$$
\begin{aligned}
& A_{k}(x), k=3, \cdots, n, \\
& B_{k}(x), k=2, \cdots, n-1, \text { and } \\
& G_{k}(x)=A[\{1, \cdots, k\},\{3, \cdots, k+2\}], k=1, \cdots, n-2,
\end{aligned}
$$


has positive determinant since the determinants of these matrices comprise all of the contiguous minors of $A$ involving $x$. By Theorem 4.7 (in the appendix) there exists an $x>0$ such that $B_{n-1}(x) \in T P_{n-2}$ is singular. Therefore, since $B_{k}(x)$, $k=2, \cdots, n-2$, and $G_{k}(x), k=1, \cdots, n-2$, are submatrices of $B_{n-1}(x)$ we have $\operatorname{det} B_{k}(x)>0, k=2, \cdots, n-2$, and $\operatorname{det} G_{k}(x)>0, k=1, \cdots, n-2$. Let

$$
F=A[\{2, \cdots, n-1\},\{2, \cdots, n\}]=\left[f_{1}, f_{2}, \cdots, f_{n-1}\right]
$$

and $B_{n-1}(x)=\left[b_{1}, b_{2}, \cdots, b_{n-1}\right]$. By Lemma 1.2, $f_{2}=\sum_{\substack{i=1 \\ i \neq 2}}^{n-1} y_{i} f_{i}$ in which $\operatorname{sgn}\left(y_{i}\right)=$ $\operatorname{sgn}(-1)^{i-1}$ and, since $B_{n-1}(x)$ is singular and $b_{1}, b_{3}, \cdots, b_{n-1}$ are linearly independent, $b_{2}=\sum_{\substack{i=1 \\ i \neq 2}}^{n-1} y_{i} b_{i}$. Thus, if $A_{n-1}(x)=\left[b_{0}, b_{1}, b_{2}, \cdots, b_{n-2}\right]$,

$$
\begin{aligned}
\operatorname{det} A_{n-1}(x) & =\operatorname{det}\left[b_{0}, b_{1}, \sum_{\substack{i=1 \\
i \neq 2}}^{n-1} y_{i} b_{i}, b_{3}, \cdots, b_{n-2}\right] \\
& =\operatorname{det}\left[b_{0}, b_{1}, y_{n-1} b_{n-1}, b_{3}, \cdots, b_{n-2}\right] \\
& =(-1)^{n-4} y_{n-2} \operatorname{det}\left[b_{0}, b_{1}, b_{3}, \cdots, b_{n-2}, b_{n-1}\right] \\
& =\left|y_{n-2}\right| \operatorname{det}\left[b_{0}, b_{1}, b_{3}, \cdots, b_{n-2}, b_{n-1}\right] \\
& >0
\end{aligned}
$$

By Sylvester's identity (2), it follows that $\operatorname{det} A=\operatorname{det} A_{n}(x)>0$ and $\operatorname{det} A_{k}(x)>0$, $k=n-2, \cdots, 3$. By decreasing $x$, det $B_{n-1}(x)$ becomes positive and we obtain a TP completion of $A$.

Case $4(s, t)=(2,2)$ : Notice that it suffices to show that there is an $x>0$ such that each of

$$
\begin{aligned}
& A_{k}(x), k=2, \cdots, n, \\
& B_{k}(x), k=2, \cdots, n-1, \\
& C_{k}(x), k=2, \cdots, n-1, \text { and } \\
& D_{k}(x), k=2, \cdots, n-1,
\end{aligned}
$$

has positive determinant since the determinants of each of these submatrices comprise all of the contiguous minors involving $x$. By Theorem 4.7, there exists an $x_{B}>0$ (resp. $x_{C}>0$ ) such that $B_{n-1}\left(x_{B}\right)$ (resp. $C_{n-1}\left(x_{C}\right)$ ) is singular and lies in $T P_{n-2}$. Without loss of generality assume $x_{B}=\min \left\{x_{B}, x_{C}\right\}$. Thus, $\operatorname{det} B_{k}\left(x_{B}\right)>0$, $k=2, \cdots, n-2$, and $\operatorname{det} C_{n-1}\left(x_{B}\right) \geq 0$. Since

$$
B_{n-1}\left(x_{B}\right)=\left[\begin{array}{cc}
* & * \\
D_{n-2}\left(x_{B}\right) & *
\end{array}\right],
$$

we have that $\operatorname{det} D_{n-2}\left(x_{B}\right)>0$ and hence, by Lemma $2.1, \operatorname{det} D_{k}\left(x_{B}\right)>0$, $k=1, \cdots, n-2$. Now

$$
C_{k}\left(x_{B}\right)=\left[\begin{array}{cc}
C_{k-1}\left(x_{B}\right) & * \\
* & *
\end{array}\right]=\left[\begin{array}{cc}
* & * \\
D_{k-1}\left(x_{B}\right) & *
\end{array}\right]
$$


$k=n-1, \cdots, 3$. So we can apply Sylvester's identity (2) repeatedly to obtain $\operatorname{det} C_{k}\left(x_{B}\right)>0, k=2, \cdots, n-2$.

We next show that $\operatorname{det} A_{k}\left(x_{B}\right)>0, k=2, \cdots, n$. Let $F=A[\{1,3, \cdots, n-1\},\{2, \cdots, n\}]=\left[f_{1}, f_{2}, \cdots, f_{n-1}\right]$ and let

$$
B_{n-1}\left(x_{B}\right)=A\left(x_{B}\right)[\{1,2, \ldots, n-1\},\{2,3, \ldots, n\}]=\left[b_{1}, b_{2}, \cdots, b_{n-1}\right] .
$$

By Lemma 1.2, $f_{1}=\sum_{i=2}^{n-1} y_{i} f_{i}$ in which $\operatorname{sgn}\left(y_{i}\right)=\operatorname{sgn}(-1)^{i}$ and since $B_{n-1}\left(x_{B}\right)$ is singular and $b_{2}, b_{3}, \cdots, b_{n-1}$ are linearly independent, $b_{1}=\sum_{i=2}^{n-1} y_{i} b_{i}$. Since $A_{n-1}\left(x_{B}\right)=\left[b_{0}, b_{1}, \cdots, b_{n-2}\right]$ we have

$$
\begin{aligned}
\operatorname{det} A_{n-1}\left(x_{B}\right) & =\operatorname{det}\left[b_{0}, \sum_{i=2}^{n-1} y_{i} b_{i}, b_{2}, \cdots, b_{n-2}\right] \\
& =\operatorname{det}\left[b_{0}, y_{n-1} b_{n-1}, b_{2}, \cdots b_{n-2}\right] \\
& =(-1)^{n-3} y_{n-1} \operatorname{det}\left[b_{0}, b_{2}, \cdots, b_{n-2}, b_{n-1}\right] \\
& =\left|y_{n-1}\right| \operatorname{det}\left[b_{0}, b_{2}, \cdots, b_{n-2}, b_{n-1}\right] \\
& >0 .
\end{aligned}
$$

It then follows from Sylvester's identity (2) that $\operatorname{det} A\left(x_{B}\right)=\operatorname{det} A_{n}\left(x_{B}\right)>0$ and since, for $k=n-1, \cdots, 3$,

$$
A_{k}=\left[\begin{array}{cc}
A_{k-1} & * \\
* & *
\end{array}\right]=\left[\begin{array}{cc}
* & B_{k-1} \\
* & *
\end{array}\right]=\left[\begin{array}{cc}
* & * \\
C_{k-1} & *
\end{array}\right]=\left[\begin{array}{cc}
* & * \\
* & D_{k-1}
\end{array}\right],
$$

we can repetitively apply Sylvester's identity (2) to obtain $\operatorname{det} A_{k}\left(x_{B}\right)>0$, $k=2, \cdots, n-1$.

To show that $\operatorname{det} D_{n-1}\left(x_{B}\right)>0$, proceed as follows. Let

$$
H=A[\{1, \cdots, n-1\},\{3, \cdots, n\}]=\left[\begin{array}{c}
H_{1} \\
H_{2} \\
\vdots \\
H_{n-1}
\end{array}\right] .
$$

By the row version of Lemma $1.2, H_{2}=\sum_{\substack{i=1 \\ i \neq 2}}^{n-1} y_{i} H_{i}$ in which $\operatorname{sgn}\left(y_{i}\right)=\operatorname{sgn}(-1)^{i-1}$. Since $B_{n-1}\left(x_{B}\right)=\left[\begin{array}{c}\beta_{1} \\ \beta_{2} \\ \vdots \\ \beta_{n-1}\end{array}\right]$ and $\beta_{1}, \beta_{3}, \cdots, \beta_{n-1}$ are linearly independent, $\beta_{2}=\sum_{\substack{i=1 \\ i \neq 2}}^{n-1} y_{i} \beta_{i}$. Now 


$$
D_{n-1}\left(x_{B}\right)=\left[\begin{array}{c}
\beta_{2} \\
\beta_{3} \\
\vdots \\
\beta_{n-1} \\
\beta_{n}
\end{array}\right]
$$

and hence

$$
\operatorname{det} D_{n-1}\left(x_{B}\right)=\operatorname{det}\left[\begin{array}{c}
\sum_{\substack{i=1 \\
i \neq 2}}^{n-1} y_{i} \beta i \\
\beta_{3} \\
\vdots \\
\beta_{n-1} \\
\beta_{n}
\end{array}\right]=\operatorname{det}\left[\begin{array}{c}
y_{1} \beta_{1} \\
\beta_{3} \\
\vdots \\
\beta_{n-1} \\
\beta_{n}
\end{array}\right]=y_{1} \operatorname{det}\left[\begin{array}{c}
\beta_{1} \\
\beta_{3} \\
\vdots \\
\beta_{n}
\end{array}\right]>0 .
$$

Now by decreasing $x_{B} \operatorname{det} B_{n-1}(x)$ and $\operatorname{det} C_{n-1}(x)$ become positive and we obtain a TP completion of $A$. If $(s, t)=(2,1)$ or $(3,1)$, then $A$ is completable to a TP matrix by reversing the indices. Thus, $A$ is completable to a TP matrix if $s+t \leq 4$. And by reversing the indices $A$ is completable to a TP matrix if $s+t \geq 2 n-2$.

Lemma 2.3. Let $A$ be an m-by-n TP matrix. Then there exists a positive vector $x \in \mathbb{R}^{n}$ such that the augmented matrix $[A \mid x]$ is an m-by-(n+1)TP matrix. Similarly, there exist positive vectors $u, v, w$ such that $[u \mid A],\left[\begin{array}{c}A \\ v\end{array}\right]$, and $\left[\begin{array}{c}w \\ A\end{array}\right]$ are all $T P$.

Proof. Consider a vector $x=\left(x_{1}, \cdots, x_{n}\right)^{T} \in \mathbb{R}^{n}$ and the augmented matrix $[A \mid x]$. The idea of the proof is to choose the $x_{i}$ 's sequentially so that $[A \mid x][\{1, \cdots, i\},\{1, \cdots, n+1\}]$ is TP. Choose $x_{1}>0$ arbitrarily. Choose $x_{2}>0$ large enough so that $[A \mid x][\{1,2\},\{1, \cdots, n+1\}]$ is TP. This is possible since $x_{2}$ enters positively into each minor that contains $x_{2}$. Continue this argument inductively. The latter part follows by analogous arguments.

Lemma 2.4. Let $A$ be an $m$-by-n partial TP matrix. Then there exist positive vectors $x, u, v, w$ such that $[A \mid x],[u \mid A],\left[\begin{array}{c}A \\ v\end{array}\right]$, and $\left[\begin{array}{c}w \\ A\end{array}\right]$ are all partial TP.

Proof. As before the idea is to choose the $x_{i}$ 's sequentially. First choose $x_{1}>0$ arbitrarily. Choose $x_{2}>0$ large enough so that all fully specified minors that involve $x_{2}$ are positive. Observe that such an $x_{2}$ exists by Lemma 2.3. Continue this argument inductively. The cases $[u \mid A],\left[\begin{array}{c}A \\ v\end{array}\right]$, and $\left[\begin{array}{c}w \\ A\end{array}\right]$ follow analogously.

Theorem 2.5. Let $A$ be an $m$-by-n partial TP matrix with $3 \leq m \leq n$ and with $x$, the only unspecified entry, lying in the $(s, t)$ position. If $s+t \leq 4$ or $s+t \geq m+n-2$, then $A$ is completable to a TP matrix.

Proof. If $s+t \leq 4$, we can apply Lemma 2.4 and obtain an $n$-by-n partial TP matrix $A_{1}=\left[\begin{array}{l}A \\ B\end{array}\right]$ in which the unspecified entry $x$ lies in the $(s, t)$ position where $s+t \leq 4$. If $s+t \geq m+n-2$, we can apply Lemma 2.4 and obtain an $n$-by- $n$ partial TP matrix $A_{2}=\left[\begin{array}{l}C \\ A\end{array}\right]$ in which the unspecified entry $x$ lies in the $(s, t)$ position where 
$s+t \geq 2 n-2$. In either case we can apply Theorem 2.2 to obtain a completion of $A_{1}$ or $A_{2}$ and hence one of $A$ itself.

THEOREM 2.6. Let $A$ be a 2-by-n partial TP matrix with exactly one unspecified entry. Then $A$ is completable to a TP matrix.

Proof. By reversing the indices we may assume without loss of generality that

$$
A=\left[\begin{array}{cccccccc}
a_{1} & a_{2} & \cdots & a_{k-1} & x & a_{k+1} & \cdots & a_{n} \\
b_{1} & b_{2} & \cdots & b_{k-1} & b_{k} & b_{k+1} & \cdots & b_{n}
\end{array}\right] .
$$

Since $A$ is partial TP it follows that $\frac{a_{i}}{b_{i}}>\frac{a_{j}}{b_{j}}$ for $i<j$ and distinct from $k$. Then, if we choose $x$ such that $a_{k-1} / b_{k-1}>x / b_{k}>a_{k+1} / b_{k+1}$, the resulting completion of $A$ is TP. $\square$

Lemma 2.7. Let $A$ be a 3-by-4 partial TP matrix with exactly one unspecified entry. Then $A$ is completable to a TP matrix.

This result simply follows from Theorem 2.5.

THEOREM 2.8. Let $A$ be a 3-by-n partial TP matrix with exactly one unspecified entry, $n \geq 3$. Then $A$ is completable to a TP matrix.

Proof. By Fekete's criterion (see Theorem 1.1) it suffices to prove the theorem for $n=5$. To see this, suppose the unspecified entry in $A$ is in column $k$. If a value for the unspecified entry can be chosen so that $A[\{1,2,3\},\{k-2, k-1, k\}]$, $A[\{1,2,3\},\{k-1, k, k+1\}]$, and $A[\{1,2,3\},\{k, k+1, k+2\}]$ (if $k=1,2, n-1$, or $n$, then consider only the submatrices above that apply) are all TP, then it follows that every contiguous minor of $A$ is positive. Thus, by Fekete's criterion, $A$ is TP. Observe that in this case it was enough to complete the submatrix of $A$, namely $A[\{1,2,3\},\{k-2, k-1, k, k+1, k+2\}]$, consisting of at most 5 columns. Hence, we can assume $n=5$. So suppose $A$ is a 3 -by-5 partial TP matrix with exactly one unspecified entry $x$. By Theorem 2.5 and Lemma 2.7 , we need only consider the case in which $x$ lies in the $(2,3)$ position. In this case there is $x_{m}>0$ such that $A\left(x_{m}\right)[\{1,2,3\},\{2,3,4\}]$ is singular and $\mathrm{TP}_{2}$ (by decreasing its $(2,2)$ entry which is the $(2,3)$ entry of $A$, see Theorem 4.3). Let $F=A[\{1,3\},\{2,3,4\}]=\left[f_{1}, f_{2}, f_{3}\right]$. Then $f_{2}=y_{1} f_{1}+y_{3} f_{3}$ where $\operatorname{sgn}\left(y_{1}\right)=\operatorname{sgn}\left(y_{3}\right)$ is positive by Lemma 1.2. Thus, $A\left(x_{m}\right)[\{1,2,3\},\{2,3,4\}]=\left[g_{1}, g_{2}, g_{3}\right]$ in which $g_{2}=y_{1} g_{1}+y_{3} g_{3}$ and so

$$
\begin{aligned}
\operatorname{det} A\left(x_{m}\right)[\{1,2,3\}] & =\operatorname{det}\left[g_{0}, g_{1}, g_{2}\right]=\operatorname{det}\left[g_{0}, g_{1}, y_{1} g_{1}+y_{3} g_{3}\right] \\
& =y_{3} \operatorname{det}\left[g_{0}, g_{1}, g_{3}\right]>0 .
\end{aligned}
$$

Similarly,

$$
\operatorname{det} A\left(x_{m}\right)[\{3,4,5\}]=\operatorname{det}\left[g_{2}, g_{3}, g_{4}\right]=y_{1} \operatorname{det}\left[g_{1}, g_{3}, g_{4}\right]>0 \text {. }
$$

Since these two 3 -by-3 minors and all 2-by-2 minors are positive, we can increase $x_{m}$ and obtain a TP completion of $A$.

Lemma 2.9. There is a 4-by-4 partial TP matrix with the $(1,4)($ or $(4,1))$ entry unspecified that has no TP completion.

Proof. Consider the partial TP matrix 


$$
A=\left[\begin{array}{cccc}
100 & 100 & 40 & x \\
40 & 100 & 100 & 40 \\
20 & 80 & 100 & 100 \\
3 & 20 & 40 & 100
\end{array}\right]
$$

In order for the determinant itself to be positive, we must have $x<-1144 / 14$. Since we must also have $x>0$, we see that $A$ is not completable to a TP matrix. $\square$

Lemma 2.10. There is a 4-by-4 partial TP matrix with the $(2,3)($ or $(3,2))$ entry unspecified that has no TP completion.

Proof. Consider the partial TP matrix

$$
A=\left[\begin{array}{cccc}
1000 & 10 & 10 & 10 \\
20 & 9 & x & 10 \\
2 & 1 & 9 & 10 \\
1 & 2 & 40 & 1000
\end{array}\right]
$$

For $\operatorname{det} A[\{1,2\},\{3,4\}]$ to be positive we must have $x<1$. But for $\operatorname{det} A[\{2,3,4\},\{1,2,3\}]$ to be positive we must have $x>199 / 3$. Hence $A$ has no TP completion. $\square$

TheOREM 2.11. (Main Result) Let $A$ be an m-by-n partial TP matrix in which $4 \leq m \leq n$ and in which the only unspecified entry lies in the $(s, t)$ position. Any such $A$ has a TP completion if and only if $s+t \leq 4$ or $s+t \geq m+n-2$.

Proof. Sufficiency follows from Theorem 2.5. To prove necessity assume that $5 \leq s+t \leq m+n-3$. Notice that if an $m$-by- $n$ matrix has this property, then there is a 4 -by- 4 submatrix such that the $(s, t)$ position of the matrix corresponds to the $(1,4),(4,1),(2,3)$, or $(3,2)$ position of that submatrix. Without loss of generality, assume that the $(s, t)$ position of the matrix corresponds to the $(1,4)$ position of the submatrix. By Lemma 2.9 there is a 4 -by-4 partial TP matrix with its only unspecified entry in the $(1,4)$ position that has no TP completion. Using Lemma 2.4 and by the preceding remarks, we can augment this matrix with positive rows/columns so that we obtain an $m$-by- $n$ partial TP matrix $A$ whose only unspecified entry lies in the $(s, t)$ position. Since the original matrix is a partial submatrix of $A$ that has no TP completion, $A$ itself has no TP completion.

3. Further Discussion. For $3 \leq m, n \leq 4$ we consider partial $m$-by- $n$ TP matrices with exactly two unspecified entries. We note that completing a 2 -by- $n$ partial TP matrix with two unspecified entries follows easily from Theorem 2.6. In the case when $\min \{m, n\}=3$ all such completable patterns have been characterized in [3]. For example, all partial 3-by-3 TP matrices with exactly two unspecified entries are completable except for the following four patterns:

$$
\left[\begin{array}{lll}
x & ? & x \\
? & x & x \\
x & x & x
\end{array}\right],\left[\begin{array}{lll}
x & x & x \\
x & x & ? \\
x & ? & x
\end{array}\right],\left[\begin{array}{lll}
x & ? & x \\
x & x & ? \\
x & x & x
\end{array}\right] \text {, and }\left[\begin{array}{ccc}
x & x & x \\
? & x & x \\
x & ? & x
\end{array}\right]
$$


Similarly, when $m=3$ and $n=4$, Table 1 contains the positions of the pairs of unspecified entries for which there is no completion in general. Observe that it is sufficient to only consider the six entries $(1,1)$ through $(2,2)$ since the conclusions for the remaining six entries follows by reversal of indices; see also [3].

\begin{tabular}{c|l}
$(1,1)$ & None \\
$(1,2)$ & $(2,1),(2,3),(3,1),(3,3)$ \\
$(1,3)$ & $(2,2),(2,4)$ \\
$(1,4)$ & $(2,3),(3,3)$ \\
$(2,1)$ & $(1,2),(3,2)$ \\
$(2,2)$ & $(1,3),(3,1),(3,3)$ \\
\multicolumn{2}{|c}{ TABLE 1 }
\end{tabular}

We begin the 4-by-4 case with some observations that aid in the classification of the completable (and hence non-completable) pairs of unspecified entries. Suppose $A$ is a partial TP matrix with exactly two unspecified entries in positions $(p, q)$ and $(s, t)$. The first observation concerns the case when either $p=s$ or $q=t$, i.e., the unspecified entries occur in the same row or the same column. In this case $A$ is always completable to a TP matrix. The idea of the proof is as follows. By Theorem 2.11, we know that the only unspecified positions in a 4-by-4 partial TP matrix that do not guarantee a TP completion are $(1,4),(2,3),(3,2)$, and $(4,1)$. Since either $p=s$ or $q=t$ at least one of the positions $(p, q)$ or $(s, t)$ is not among the list of four "bad" positions listed above. Without loss of generality assume that $p=s$. Then delete the column that corresponds to the entry that is not in the above list, and complete the remaining 4-by-3 partial TP matrix to a TP matrix (which follows from Theorem 2.8). Observe that now $A$ is a partial TP matrix with only one unspecified entry. Since this remaining unspecified entry is in a "good" position, it follows that we can complete $A$ to a TP matrix.

The next observation deals with the case when one of the unspecified entries is in the $(1,1)$ (or $(4,4)$ ) position and the other unspecified entry is in a "good" position, i.e., satisfies one of the conditions in Theorem 2.11. Since the $(1,1)$ (or $(4,4)$ ) entry enters positively into every minor that it is contained in, it follows that we can specify a large enough value for this entry so that $A$ is a partial TP matrix with only one unspecified entry. Then $A$ may be completed to TP matrix since the remaining unspecified entry is in a "good" position. We note here that this observation can be extended in the general case as follows. If $A$ is an $m$-by- $n$ partial TP matrix with exactly two unspecified entries with one in the $(1,1)$ (or $(m, n)$ ) position and the other in a "good" position (as defined by Theorem 2.11), then $A$ is completable to a TP matrix. (The proof in this general case is identical to the proof in the 4 -by-4 case.)

Finally, suppose the unspecified entries occur in positions $(p, q)$ and $(s, t)$ where either $|p-s|=3$, or $|q-t|=3$. Then $A$ is completable to a TP matrix except in the case when $(p, q)=(1,4)$ and $(s, t)=(4,1)$ (or vice-versa). First assume that 
$(p, q)=(1,4)$ and $(s, t)=(4,1)$, and consider the following example. Let

$$
A(x, y)=\left[\begin{array}{llll}
1 & 1 & .4 & x \\
.4 & 1 & 1 & .4 \\
.2 & .8 & 1 & 1 \\
y & .2 & .4 & 1
\end{array}\right]
$$

see also [14]. Then it is easy to check that every fully specified minor of $A(x, y)$ is positive. However,

$$
\operatorname{det} A(x, y)=-.0016-.008 x-.328 y-.2 x y,
$$

Hence $\operatorname{det} A(x, y)<0$ for $x$ and $y$ positive. On the other hand assume that $(p, q) \neq$ $(1,4)$ or $(s, t) \neq(4,1)$. Then since either $|p-s|=3$, or $|q-t|=3$ it follows that at least one of the unspecified entries is in a "good" position. Without loss of generality assume that $q \geq t$ and that $q-t=3$. Hence $q=4$ and $t=1$. Now delete the column of the unspecified entry that is in a good position. By Theorem 2.8, we may complete the remaining 4-by-3 matrix to a TP matrix. Now consider the 4 -by-4 matrix $A$ with only one unspecified entry. If we can show that $A$ is a partial TP matrix, then we are done, since the remaining unspecified entry is in a "good" position. The reason that $A$ (with only one unspecified entry) is partial TP is because since $|q-t|=3$ all of the fully specified minors of $A$ based on contiguous index sets are positive. Hence, by Fekete's criterion, $A$ is a partial TP matrix.

Using the above three observations the pairs of positions for the unspecified entries not ruled out are contained in Table 2 .

$$
\begin{array}{c|l}
(1,1) & (2,3),(3,2) \\
(1,2) & (2,1),(2,3),(2,4),(3,1),(3,3),(3,4) \\
(1,3) & (2,1),(2,2),(2,4),(3,1),(3,2),(3,4) \\
(1,4) & (2,2),(2,3),(3,2),(3,3) \\
(2,2) & (1,3),(1,4),(3,1),(3,3),(3,4),(4,1),(4,3) \\
(2,3) & (1,1),(1,2),(1,4),(3,1),(3,2),(3,4),(4,1),(4,2),(4,4) \\
& \text { TABLE 2 }
\end{array}
$$

As before we only have to consider the six entries: $(1,1),(1,2),(1,3),(1,4),(2,2)$, and $(2,3)$, since the class TP is closed under transposition and reversal of indices. In fact the list in Table 2 can be even further refined as some of the above cases follow from others by transposition and/or reversal of indices; see Table 3.

As we shall see all but one of the above (namely, the pair $\{(1,4),(3,2)\}$ ) pairs are not in general completable. We begin by first ruling out the pairs that follow from the pairs of non-completable entries in the 3 -by- 3 and 3 -by- 4 cases. For example, suppose the unspecified entries are in the $(1,2)$ and $(2,1)$ positions. There exists a 3 -by-3 TP matrix with unspecified entries in those positions that has no TP completion, e.g., $\left[\begin{array}{ccc}3 & x & 2 \\ y & 3 & 1 \\ 3 & 4 & 3\end{array}\right]$. We can border this 3-by-3 partial TP matrix (by Lemma 2.4) to 
$(1,1) \mid(2,3)$

$(1,2) \quad(2,1),(2,3),(2,4),(3,1),(3,3),(3,4)$

$(1,3) \quad(2,2),(2,4),(3,1),(3,2)$,

$(1,4) \quad(2,2),(2,3),(3,2),(3,3)$

$(2,2) \quad(3,3)$

$(2,3) \quad(3,2),(3,4)$

TABLE 3

obtain a 4-by-4 partial TP matrix that has no TP completion. Similar arguments can be applied (using both the 3 -by-3 and 3 -by- 4 results) to rule out the pairs: $\{(1,2)$, $(2,3)\},\{(1,2),(3,1)\},\{(1,2),(3,3)\},\{(1,3),(2,2)\},\{(1,3),(2,4)\},\{(1,4),(2,3)\},\{(1,4)$, $(3,3)\},\{(2,2),(3,3)\},\{(2,3),(3,2)\},\{(2,3),(3,4)\}$. For the remaining cases we consider examples of 4 -by-4 partial TP matrices.

1. $(1,1),(2,3)$ : Let

$$
A=\left[\begin{array}{cccc}
x & 1 & 1 & 1 \\
2 & .9 & y & 1 \\
.2 & .1 & .9 & 1 \\
.2 & .2 & 4 & 100
\end{array}\right]
$$

Then $A$ is a partial TP matrix. However, $\operatorname{det} A[\{2,3,4\},\{1,2,3\}]>0$ if and only if $y>6.3$. But for $y>6.3, \operatorname{det} A[\{1,2\},\{3,4\}]<0$. Hence $A$ has no TP completion.

2. $(1,2),(2,4)$ : Let

$$
A=\left[\begin{array}{cccc}
3.21 & x & 2 & 1 \\
2.3 & 2.1 & 2 & y \\
2.1 & 2 & 2 & 2 \\
1 & 1 & 2 & 3
\end{array}\right]
$$

Then $A$ is a partial TP matrix. However, $A$ has no TP completion since $\operatorname{det} A[\{1,2\}]>0 \Leftrightarrow x<2.931$, and $\operatorname{det} A[\{1,3,4\},\{2,3,4\}]>0 \Leftrightarrow x>3$.

3. $(1,2),(3,4)$ : Let

$$
A=\left[\begin{array}{cccc}
3 & x & 2 & 1 \\
2 & 2.1 & 2 & 2 \\
1 & 2 & 2 & y \\
.4 & 1 & 2 & 4
\end{array}\right] .
$$

Then $A$ is a partial TP matrix. However, $A$ has no TP completion since $\operatorname{det} A[\{2,3,4\}]>0 \Leftrightarrow y<2.18$, and $\operatorname{det} A[\{1,2,3\},\{1,3,4\}]>0 \Leftrightarrow y>3$.

4. $(1,3),(3,1)$ : Let

$$
A=\left[\begin{array}{cccc}
2.5 & 2 & x & 1 \\
2 & 2 & 1.21 & 2 \\
y & 2 & 1.5 & 2.5 \\
.3 & 2 & 3 & 10
\end{array}\right]
$$


Then $A$ is a partial TP matrix. However, $A$ has no TP completion since $\operatorname{det} A[\{1,2,3\},\{1,2,4\}]>0 \Leftrightarrow y>1.75$, and $\operatorname{det} A[\{2,3,4\},\{1,2,3\}]>0 \Leftrightarrow$ $y<1.725$.

5. $(1,3),(3,2)$ : Let

$$
A=\left[\begin{array}{cccc}
3 & 2 & x & 1 \\
2 & 2 & 1.1 & 2 \\
.3 & y & 1 & 2 \\
1 & 5 & 4 & 10
\end{array}\right]
$$

Then $A$ is a partial TP matrix. However, $A$ has no TP completion since $\operatorname{det} A[\{2,3,4\},\{1,2,3\}]>0 \Leftrightarrow y>1.268$, and $\operatorname{det} A[\{1,2,3\},\{1,2,4\}]>0 \Leftrightarrow$ $y<1.15$.

6. $(1,4),(2,2)$ : Let

$$
A=\left[\begin{array}{cccc}
10 & 2 & 3 & x \\
3.3 & y & 2 & 1 \\
2.1 & 2 & 2 & 2 \\
1 & 1 & 2 & 3
\end{array}\right] .
$$

Then $A$ is a partial TP matrix. However, $A$ has no TP completion since $\operatorname{det} A[\{2,3,4\}]>0 \Leftrightarrow y>3$, and $\operatorname{det} A[\{1,2\},\{2,3\}]>0 \Leftrightarrow y<4 / 3$.

The only pair remaining is $\{(1,4),(3,2)\}$. As we shall see, this pair is actually a completable pair. However, the argument presented below is a little long and requires a lemma (which provides a necessary and sufficient condition for the completability of the $(1,4)$ entry in a 4 -by-4 matrix) which we now state and prove.

LEMma 3.1. Let $A$ be a partial TP matrix with the $(1,4)$ (or $(4,1))$ entry unspecified, and let $A(x)$ denote the matrix where $x$ is a value that is specified for the $(1,4)$ (or $(4,1))$ entry of $A$. Then $A$ has a TP completion if and only if $\operatorname{det} A(0)>0$.

Proof. Observe that $\operatorname{det} A(x)=\operatorname{det} A(0)-x \operatorname{det} A[\{2,3,4\},\{1,2,3\}]$. Thus it is clear that $A$ has no TP completion if $\operatorname{det} A(0) \leq 0$. To verify that $A$ can be completed to a TP matrix in the case when $\operatorname{det} A(0)>0$ it is enough to show that there exists an $x>0$ such that $\operatorname{det} A(x)>0, \operatorname{det} A(x)[\{1,2,3\},\{2,3,4\}]>0$, and $\operatorname{det} A(x)[\{1,2\},\{3,4\}]>0$, by Fekete's criterion. Note that such an $x>0$ exists if

$$
\frac{-\operatorname{det} A(0)[\{1,2,3\},\{2,3,4\}]}{\operatorname{det} A[\{2,3\}]}<\min \left\{\frac{a_{13} a_{24}}{a_{23}}, \frac{\operatorname{det} A(0)}{\operatorname{det} A[\{2,3,4\},\{1,2,3\}]}\right\} .
$$

Now observe that by Sylvester's identity (2)

$$
\begin{aligned}
\operatorname{det} A(0)[\{1,2,3\},\{2,3,4\}]= & \\
& =\frac{\operatorname{det} A[\{1,2\},\{2,3\}] \operatorname{det} A[\{2,3\},\{3,4\}]-\operatorname{det} A[\{2,3\}] a_{13} a_{24}}{a_{23}} \\
& >\frac{-\operatorname{det} A[\{2,3\}] a_{13} a_{24}}{a_{23}},
\end{aligned}
$$

or

$$
\frac{-\operatorname{det} A(0)[\{1,2,3\},\{2,3,4\}]}{\operatorname{det} A[\{2,3\}]}<\frac{a_{13} a_{24}}{a_{23}}
$$


Similar arguments show

$$
\frac{-\operatorname{det} A(0)[\{1,2,3\},\{2,3,4\}]}{\operatorname{det} A[\{2,3\}]}<\frac{\operatorname{det} A(0)}{\operatorname{det} A[\{2,3,4\},\{1,2,3\}]} .
$$

This completes the proof.

We are now in a position to prove that the pair $\{(1,4),(3,2)\}$ is a completable pair.

Proposition 3.2. Let $A$ be a 4-by-4 partial TP matrix with the $(1,4)$ and $(3,2)$ entries unspecified. Then A may be completed to a TP matrix.

Proof. Let

$$
A=\left[\begin{array}{cccc}
a_{11} & a_{12} & a_{13} & x \\
a_{21} & a_{22} & a_{23} & a_{24} \\
a_{31} & y & a_{33} & a_{34} \\
a_{41} & a_{42} & a_{43} & a_{44}
\end{array}\right] .
$$

First choose $y$ such that $A[\{2,3,4\},\{1,2,3\}]$ is singular but $\mathrm{TP}_{2}$ (such a $y$ exists by Theorem 4.3 and is unique). Write $A[\{2,3,4\},\{1,2,3,4\}]=\left[a_{1}, a_{2}, a_{3}, a_{4}\right]$, where $a_{i}$ is the $i^{\text {th }}$ column of $A[\{2,3,4\},\{1,2,3,4\}](i=1,2,3,4)$. Then by applying Lemma 1.2 to $A[\{2,3\},\{1,2,3\}]$ and $A[\{3,4\},\{1,2,3\}]$ we have that $a_{2}=\alpha_{1} a_{1}+\alpha_{3} a_{3}$, where $\alpha_{1}, \alpha_{3}>0$. We claim that all minors of $A[\{2,3,4\},\{1,2,3,4\}]$ are positive except, of course, $A[\{2,3,4\},\{1,2,3\}]$. The 2 -by-2 minors are easily verified to be positive. For the 3 -by-3 minors consider $A[\{2,3,4\},\{1,2,4\}]$, for example. Observe, that

$$
\begin{aligned}
\operatorname{det} A[\{2,3,4\},\{1,2,4\}] & =\operatorname{det}\left[a_{1}, a_{2}, a_{4}\right]=\alpha_{1} \operatorname{det}\left[a_{1}, a_{1}, a_{4}\right]+\alpha_{3} \operatorname{det}\left[a_{1}, a_{3}, a_{4}\right] \\
& =\alpha_{3} \operatorname{det}\left[a_{1}, a_{3}, a_{4}\right]>0 .
\end{aligned}
$$

Similarly, all the minors of $A[\{1,2,3,4\},\{1,2,3\}]$ are positive except for $A[\{2,3,4\},\{1,2,3\}]$. Since $\operatorname{det} A[\{2,3,4\},\{1,2,3\}]=0$ and $A$ is partial TP it follows that for any value of $x, \operatorname{det} A>0$. Moreover, we can increase $y$ a small amount so that $\operatorname{det} A[\{2,3,4\},\{1,2,3\}]>0$, all other fully specified minors of $A$ are positive, and $\operatorname{det} A>0$ when $x=0$. Hence by Lemma 3.1 we can complete $A$ to a TP matrix. $\square$

Table 4 contains a complete list of all non-completable pairs for a 4 -by-4 partial TP matrix.

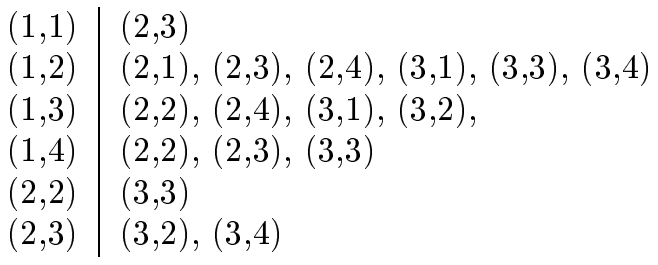

TABLE 4

For larger values of $m$ and $n$, a complete classification of all possible pairs of completable entries seems to be difficult and remains open. 
We close this section we some final remarks on other general patterns that yield a TP completion. Recall that the $(1,1)$ and $(m, n)$ can be increased without bound in a TP matrix and preserve the property of being TP. This fact proves that the following pattern always has a TP completion. Let $A$ be a partial TP matrix whose unspecified entries have the following property: if $a_{i j}=$ ?, then either $a_{s t}=$ ?, for $s \leq i$ and $t \leq j$ or $a_{s t}=$ ?, for $s \geq i$ and $t \geq j$. For example, suppose $A$ is a partial TP matrix such that if $a_{i j}=$ ?, then $a_{s t}=$ ?, for $s \leq i$ and $t \leq j$, Then begin completing $A$ by choosing a value for the bottom rightmost unspecified entry large enough so that the fully specified submatrix below and to the right is TP, and continue this process by moving to the left until the entire row has been completed. Repeat this process by moving one row up and continue. Observe that at each stage in this process there is always a choice for the unspecified entry since it occurs in the " $(1,1)$ " position of the submatrix that is to be completed. Finally, in [16] it is shown that one can always insert a row or a column into a TP matrix and remain TP.

4. Appendix: Single Entry Perturbations of Totally Positive matrices. It is not difficult to prove that if we increase the $(1,1)$ or the $(m, n)$ entry of an $m$-by-n totally nonnegative (positive) matrix, then the resulting matrix is totally nonnegative (positive). We wish to investigate further which entries of a totally nonnegative (positive) matrix may be perturbed (i.e., increased or decreased) so that the result is a totally nonnegative (positive) matrix. These issues have already been addressed for other positivity classes of matrices, for example, if $A$ is an $n$-by- $n$ positive semidefinite, $M_{-}, P_{-}$, or inverse $M$-matrix, then $A+D$ ( $D$ a nonnegative diagonal matrix) is a positive semidefinite, $M-, P$-, or inverse $M$-matrix, respectively; see [10, 11]. Recall that $E_{i j}$ denotes the $n$-by-n $(i, j)^{t h}$ standard basis matrix, i.e., the matrix whose $(i, j)^{t h}$ entry is 1 and whose remaining entries are zero. Suppose $A$ is an $n$-by- $n$ matrix. Then $\operatorname{det}\left(A-t E_{11}\right)=\operatorname{det} A-t \operatorname{det} A(\{1\})$. Therefore, if $\operatorname{det} A(\{1\}) \neq 0$, then $\operatorname{det}\left(A-t E_{11}\right)=0$, when $t=\operatorname{det} A / \operatorname{det} A(\{1\})$. Consider the following lemma.

Lemma 4.1. Let $A$ be an $n$-by-n totally nonnegative matrix with $\operatorname{det} A(\{1\}) \neq 0$. Then $A-x E_{11}$ is totally nonnegative for all $x \in[0, \operatorname{det} A / \operatorname{det} A(\{1\})]$.

Proof. Firstly, observe that for every value $x \in[0, \operatorname{det} A / \operatorname{det} A(\{1\})], \operatorname{det}(A-$ $\left.x E_{11}\right) \geq 0$. Recall that $A$ admits a $U L$-factorization (see [1]) into totally nonnegative matrices. Partition $A$ as follows,

$$
A=\left[\begin{array}{ll}
a_{11} & a_{12}^{T} \\
a_{21} & A_{22}
\end{array}\right]
$$

where $a_{11}$ is 1 -by- 1 and $A_{22}=A(\{1\})$. Partition $L$ and $U$ conformally with $A$. Then

$$
\begin{aligned}
A & =\left[\begin{array}{cc}
a_{11} & a_{12}^{T} \\
a_{21} & A_{22}
\end{array}\right]=U L \\
& =\left[\begin{array}{cc}
u_{11} & u_{12}^{T} \\
0 & U_{22}
\end{array}\right]\left[\begin{array}{cc}
l_{11} & 0 \\
l_{21} & L_{22}
\end{array}\right] \\
& =\left[\begin{array}{cc}
u_{11} l_{11}+u_{12}^{T} l_{21} & u_{12}^{T} L_{22} \\
U_{22} l_{21} & U_{22} L_{22}
\end{array}\right] .
\end{aligned}
$$


Note that if $l_{11}=0$, then $L$, and hence $A$, is singular. In this case the interval for $x$ is degenerate, and $x=0$ is the only allowed value for $x$. The desired result is trivial. Thus we assume that $l_{11}>0$. Consider the matrix $A-x E_{11}$, with $x \in$ $[0, \operatorname{det} A / \operatorname{det} A(\{1\})]$. Then

$$
\begin{aligned}
A-x E_{11} & =\left[\begin{array}{cc}
u_{11} l_{11}+u_{12}^{T} l_{21}-x & u_{12}^{T} L_{22} \\
U_{22} l_{21} & U_{22} L_{22}
\end{array}\right] \\
& =\left[\begin{array}{cc}
u_{11}-\frac{x}{l_{11}} & u_{12}^{T} \\
0 & U_{22}
\end{array}\right]\left[\begin{array}{cc}
l_{11} & 0 \\
l_{21} & L_{22}
\end{array}\right]=U^{\prime} L .
\end{aligned}
$$

To show that $A-x E_{11}$ is totally nonnegative it is enough to verify that $u_{11}-x / l_{11} \geq 0$. Since if this was the case it follows that $U^{\prime}$ is totally nonnegative and as $L$ is totally nonnegative by assumption, we have that their product, $A-x E_{11}$ is totally nonnegative. Since $l_{11}>0$ and $\operatorname{det} A(\{1\})>0$, it follows that $L$ and $U_{22}$ are nonsingular. Hence $0 \leq \operatorname{det}\left(A-x E_{11}\right)=\left(u_{11}-x / l_{11}\right) \operatorname{det} U_{22} \operatorname{det} L$, from which it follows that $u_{11}-x / l_{11} \geq 0$. $\mathrm{\square}$

Note that a similar result holds for decreasing the $(n, n)$ entry. We can extend the previous result for the class TP as follows.

THeOREM 4.2. Let $A$ be an $n$-by-n totally positive matrix. Then $A-t E_{11}$ is a $T P_{n-1}$ matrix, for all $t \in[0, \operatorname{det} A / \operatorname{det} A(\{1\})]$.

Proof. Following the proof of Lemma 4.1 we can write

$$
A-t E_{11}=\left[\begin{array}{cc}
u_{11}-\frac{t}{l_{11}} & u_{12}^{T} \\
0 & U_{22}
\end{array}\right]\left[\begin{array}{cc}
l_{11} & 0 \\
l_{21} & L_{22}
\end{array}\right]=U^{\prime} L
$$

where $U^{\prime}=U-\left(\frac{t}{l_{11}}\right) E_{11}$, and both $U, L$ are triangular TP matrices; see [1]. Observe that $u_{11} l_{11}=\operatorname{det} A / \operatorname{det} A(\{1\})$. If $u_{11}-t / l_{11}>0$, then $U^{\prime}$ and $L$ are triangular TP matrices and hence $A-t E_{11}$ is TP. So consider the case $u_{11}-t / l_{11}=0$, or equivalently, $\operatorname{det}\left(A-t E_{11}\right)=0$, or $t=\operatorname{det} A / \operatorname{det} A(\{1\})$. Let $B=A-t E_{11}$. Observe that $B[\{1,2, \ldots, n\},\{2,3, \ldots, n\}]$ and $B[\{2,3 \ldots, n\},\{1,2, \ldots, n\}]$ are TP matrices since $A$ is TP. Thus the only contiguous minors left to verify are the leading contiguous minors of $B$. Consider the submatrices $B[\{1,2, \ldots, k\}]$ for $1 \leq k<n$. Then $\operatorname{det} B[\{1,2, \ldots, k\}]=\operatorname{det} A[\{1,2, \ldots, k\}]-t \operatorname{det} A[\{2, \ldots, k\}]$. This minor is positive if and only if $\operatorname{det} A[\{1,2, \ldots, k\}]>t \operatorname{det} A[\{2, \ldots, k\}]$, which is equivalent to $\operatorname{det} A[\{1,2, \ldots, k\}] \operatorname{det} A(\{1\})>\operatorname{det} A \operatorname{det} A[\{2, \ldots, k\}]$, an example of a Koteljanskii inequality (see [11]). The only issue left to settle is whether or not equality holds for the above Koteljanskii inequality. We claim here that for a TP matrix every Koteljanskii inequality is strict. Suppose to the contrary, i.e., assume there exist two index sets $\alpha$ and $\beta$ such that $\operatorname{det} A[\alpha \cup \beta] \operatorname{det} A[\alpha \cap \beta]=\operatorname{det} A[\alpha] \operatorname{det} A[\beta]$. For simplicity, we may assume that $\alpha \cup \beta=N$, otherwise replace $A$ by $A[\alpha \cup \beta]$ in the following. By Jacobi's identity (see [10]) we have $\operatorname{det} A^{-1}\left[(\alpha \cup \beta)^{c}\right] \operatorname{det} A^{-1}\left[(\alpha \cap \beta)^{c}\right]=\operatorname{det} A^{-1}\left[\alpha^{c}\right] \operatorname{det} A^{-1}\left[\beta^{c}\right]$. Let $B=S A^{-1} S$, for $S=\operatorname{diag}(1,-1, \cdots, \pm 1)$. Then $B$ is TP and the above equation implies $\operatorname{det} B=\operatorname{det} B\left[\alpha^{c}\right] \operatorname{det} B\left[\beta^{c}\right]$. By a result in [2], $B$ is reducible, which is nonsense since $B$ is TP. Thus $A-t E_{11}$ is $\mathrm{TP}_{n-1}$, by Fekete's criterion. This completes the proof. 
An obvious next question is what other entries can be increased/decreased to the point of singularity so that the matrix is $\mathrm{TP}_{n-1}$. As it turns out decreasing the $(2,2)$ entry of a TP matrix results in a $\mathrm{TP}_{n-1}$ matrix.

Theorem 4.3. Let $A$ be an $n$-by-n totally positive matrix. Then $A-t E_{22}$ is $T P_{n-1}$ for all $t \in[0, \operatorname{det} A / \operatorname{det} A(\{2\})]$.

Proof. Using the fact that all Koteljanskii inequalities are strict it follows that all of the leading proper principal minors of $A-t E_{22}$ are positive. Consider the submatrix $B=\left(A-t E_{22}\right)[\{1,2, \ldots, n\},\{2,3, \ldots, n\}]$. To show that $B$ is TP we need only consider the contiguous minors of $B$ that involve the first and second row and first column, all other minors are positive by assumption. Let $C$ denote such a submatrix of $B$. To compute $\operatorname{det} C$, expand the determinant along the second row of $C$. Then $\operatorname{det} C=(-1)^{1+2}(-t) \operatorname{det} C(\{2\},\{1\})+\operatorname{det} A[\alpha, \beta]$, where $\operatorname{det} A[\alpha, \beta]$ is some minor of $A$. Thus $\operatorname{det} C$ is a positive linear combination of minors of $A$, and hence is positive. Therefore $B$ is TP. Similar arguments show that $\left(A-t E_{22}\right)[\{2, \ldots, n\},\{1,2, \ldots, n\}]$ is TP. This completes the proof.

A similar fact holds for decreasing the $(n-1, n-1)$ entry of a TP matrix. The next result follows directly from Theorems 4.2 and 4.3 .

Corollary 4.4. If $A$ is an $n$-by-n totally positive matrix and $i \in\{1,2, n-1, n\}$, then $A-t E_{i i}$ is $T P_{n-1}$ for all $t \in[0, \operatorname{det} A / \operatorname{det} A(\{i\})]$.

COROllary 4.5. Let $n \leq 4$. If $A$ is an $n$-by-n totally positive matrix and $1 \leq i \leq n$, then $A-t E_{i i}$ is $T P_{n-1}$ for all $t \in[0, \operatorname{det} A / \operatorname{det} A(\{i\})]$.

According to the next example we cannot decrease any other interior main diagonal entry (in general) of a TP matrix and stay $\mathrm{TP}_{n-1}$.

EXAMPLE 4.6. Consider the following matrix.

$$
A=\left[\begin{array}{ccccc}
100 & 10 & 7 / 5 & 2 & 1 \\
22 & 5 & 2 & 3 & 2 \\
3 & 1 & 1.01 & 2 & 3 \\
1 & 1 & 2 & 5 & 12 \\
1 / 2 & 2 & 5 & 15 & 50
\end{array}\right]
$$

Then $A$ is a totally positive matrix with $\operatorname{det} A / \operatorname{det} A(\{3\}) \approx .03$. However,

$$
\frac{\operatorname{det} A[\{1,2,3\},\{3,4,5\}]}{\operatorname{det} A[\{1,2\},\{4,5\}]}=.01 \text {. }
$$

Thus for $t \in(.01, .03], \operatorname{det}\left(A-t E_{33}\right)[\{1,2,3\},\{3,4,5\}]<0$, and hence $A-t E_{33}$ is not $\mathrm{TP}_{4}$.

Note that this example can be embedded into a larger example by bordering the matrix above so as to preserve the property of being TP (using Lemma 2.3, for example).

Up to this point we have only considered decreasing a single diagonal entry, and obvious next step is to consider increasing or decreasing off-diagonal entries in a TP matrix. We begin our study of perturbing off-diagonal entries by considering the $(1,2)$ entry.

TheOrem 4.7. Let $A$ be an n-by-n totally positive matrix. Then $A+t E_{12}$ is $T P_{n-1}$ for all $t \in[0, \operatorname{det} A / \operatorname{det} A(\{1\},\{2\})]$. 
Proof. Since the $(1,2)$ entry of $A$ enters negatively into $\operatorname{det} A$ we increase $a_{12}$ to $a_{12}+\operatorname{det} A / \operatorname{det} A(\{1\},\{2\})$ so that $\operatorname{det}\left(A+t E_{12}\right)=0$, where $t=\operatorname{det} A / \operatorname{det} A(\{1\},\{2\})$. Observe that the submatrix $\left(A+t E_{12}\right)[\{2, \ldots, n\},\{1,2, \ldots, n\}]$ is equal to $A[\{2, \ldots, n\},\{1,2, \ldots, n\}]$ and hence is TP. Moreover,

$\left(A+t E_{12}\right)[\{1,2, \ldots, n\},\{2, \ldots, n\}]$ is TP since we have increased the " $(1,1)$ " entry of a TP matrix. The only remaining minors to verify are the leading principal minors of $A+t E_{12}$. Observe that for $t \in[0, \operatorname{det} A / \operatorname{det} A(\{1\},\{2\})]$,

$$
\begin{aligned}
& 0 \leq \operatorname{det}\left(A+t E_{12}\right) \operatorname{det} A[\{2,3, \ldots, n-1\}] \\
& =\operatorname{det}\left(A+t E_{12}\right)[\{1,2, \ldots, n-1\}] \operatorname{det} A[\{2,3, \ldots, n\}] \\
& \quad-\operatorname{det}\left(A+t E_{12}\right)[\{1,2, \ldots, n-1\},\{2, \ldots, n\}] \operatorname{det} A[\{2, \ldots, n\},\{1,2, \ldots, n-1\}],
\end{aligned}
$$

follows by Sylvester's identity (2). Hence $\operatorname{det}\left(A+t E_{12}\right)[\{1,2, \ldots, n-1\}]>0$. Replacing $A+t E_{12}$ by $\left(A+t E_{12}\right)[\{1,2, \ldots, n-1\}]$ in the above identity yields $\operatorname{det}(A+$ $\left.t E_{12}\right)[\{1,2, \ldots, n-2\}]>0$ and so on. This completes the proof.

Corollary 4.8. If $A$ is an $n$-by-n totally positive matrix and $(i, j)=(2,1)$, $(n-1, n)$, or $(n, n-1)$, then $A+t E_{i j}$ is $T P_{n-1}$ for all $t \in[0, \operatorname{det} A / \operatorname{det} A(\{i\},\{j\})]$.

Unfortunately, this is all that can be said positively concerning increasing or decreasing off-diagonal entries. Consider the following example.

EXAMPLE 4.9. Let

$$
A=\left[\begin{array}{cccc}
13 & 33 & 31 & 10 \\
132 & 383 & 371 & 120 \\
13 & 38 & 37 & 12 \\
1 & 3 & 3 & 1
\end{array}\right]
$$

Then $\operatorname{det} A / \operatorname{det} A(\{1\},\{3\})=1$ and $\operatorname{det} A[\{1,2\},\{3,4\}] / a_{24}=1 / 12$. Thus for $t \in(1 / 12,1], \operatorname{det}\left(A-t E_{13}\right)[\{1,2\},\{3,4\}]<0$. Thus $A-t E_{13}$ is not $\mathrm{TP}_{3}$.

For the $(2,3)$ entry of a TP matrix consider the following example. Let

$$
A=\left[\begin{array}{llll}
1 & 12 & 111 & 100 \\
3 & 37 & 344 & 310 \\
3 & 37 & 356 & 321 \\
1 & 13 & 123 & 112
\end{array}\right]
$$

Then $\operatorname{det} A / \operatorname{det} A(\{2\},\{3\})=1 / 3$ and $\operatorname{det} A[\{1,2\},\{3,4\}] / a_{44}=1 / 10$. Thus for $t \in(1 / 10,1 / 3], \operatorname{det}\left(A+t E_{23}\right)[\{1,2\},\{3,4\}]<0$. Thus $A+t E_{23}$ is not $\mathrm{TP}_{3}$.

Finally, of the case of the $(1,4)$ entry we have the following example. Let

$$
A=\left[\begin{array}{cccc}
1 & 2 & 3 & 1 \\
3 & 7 & 11 & 4 \\
3 & 8 & 14 & 6 \\
1 & 3 & 6 & 4
\end{array}\right]
$$

Then $\operatorname{det} A / \operatorname{det} A(\{1\},\{4\})=1$ and $\operatorname{det} A[\{1,2\},\{3,4\}] / a_{23}=1 / 11$. Thus for $t \in(1 / 11,1], \operatorname{det}\left(A+t E_{14}\right)[\{1,2\},\{3,4\}]<0$. Thus $A+t E_{14}$ is not $\mathrm{TP}_{3}$. 
As in the case of the diagonal entries these three examples above can be embedded into a larger TP matrix (using Lemma 2.3) to show that in general there are no other

off-diagonal entries that can be increased/decreased (until the matrix is singular) and the resulting matrix lie in the class $\mathrm{TP}_{n-1}$.

\section{REFERENCES}

[1] T. Ando, Totally Positive Matrices, Linear Algebra and its Applications. 90:165-219, 1987.

[2] D. Carlson, Weakly, Sign-Symmetric Matrices and Some Determinantal Inequalities, Colloquium Mathematicum 17:123-129, 1967.

[3] K.E. DiMarco and C.R. Johnson, Totally Positive Pattern Completions, An unpublished paper from the National Science Foundation Research Experiences for Undergraduates program held at the College of William and Mary in the summer of 1998.

[4] J.H. Drew and C.R. Johnson, The completely positive and doubly nonnegative completion problems, Linear and Multilinear Algebra 44:85-92, 1998.

[5] J.H. Drew, C.R. Johnson, K.L. Karlof and K.L. Shuman, The cycle completable graphs for the completely positive and doubly nonnegative completion problems, manuscript, 1999.

[6] E.B. Dryden and C.R. Johnson, Totally Nonnegative Completions, An unpublished paper from the National Science Foundation Research Experiences for Undergraduates program held at the College of William and Mary in the summer of 1997.

[7] F.R. Gantmacher and M.G. Krein, Oszillationsmatrizen, Oszillationskerne und kleine Schwingungen Mechanischer Systeme, Akademie-Verlag, Berlin, 1960.

[8] R. Grone, C.R. Johnson, E. Sa and H. Wolkowicz, Positive definite completions of partial hermitian matrices, Linear Algebra and its Applications. 58:109-124, 1984.

[9] M. Gasca and C.A. Micchelli, Total Positivity and its Applications, Mathematics and its Applications, Vol. 359, Kluwer Academic, 1996.

[10] R. A. Horn and C. R. Johnson, Matrix Analysis, Cambridge University Press, New York, 1985.

[11] R. A. Horn and C. R. Johnson, Topics in Matrix Analysis, Cambridge University Press, New York, 1991.

[12] C.R. Johnson, Matrix completion problems: A Survey, Proceedings of Symposia in Applied Mathematics 40:171-198, 1990.

[13] C.R. Johnson and B.K. Kroschel, The combinatorially symmetric $P$-matrix completion problem, Electronic Journal of Linear Algebra 1:59-63, 1996.

[14] C.R. Johnson, B.K. Kroschel, and M. Lundquist The totally nonnegative completion problem, Fields Institute Communications, American Mathematical Society, Providence, RI, 18:97$108,1998$.

[15] C.R. Johnson and R.L. Smith, The completion problem for M-matrices and inverse M-matrices, Linear algebra and its Applications. 241-243:655-667, 1996.

[16] C.R. Johnson and R.L. Smith, Insertions in Totally Positive Matrices, in preparation.

[17] S. Karlin, Total Positivity, Vol. I, Stanford University Press, Stanford, 1968. 